\title{
An Asymptotic-Numerical Approach to the Coronal Loop Problem
}

\author{
F. Verhulst and P. A. Zegeling $\dagger$ \\ Mathematisch Instituut, Rijksuniversiteit Utrecht, PO Box 80.010, 3508 TA Utrecht, \\ The Netherlands
}

Communicated by H. Neunzert

\begin{abstract}
The coronal loop problem is characterized by mixed boundary conditions and the loop length condition, which is global. Using singular perturbation methods one can identify and construct two boundary layers at the base of the loop.

Extending this to a combined asymptotic-numerical treatment it is possible to construct two static solutions satisfying the same conditions; this unusual feature arises from the presence of the first boundary layer, which corresponds with a steep temperature gradient and an energy balance dominated by conduction and radiation losses.
\end{abstract}

\section{Introduction}

The behaviour of a hot plasma, confined to a magnetic loop as observed in the solar corona, has been the subject of various modelling attempts and explicit calculations. In this note we extend and correct the study by Pakkert et al. [2] where a model has been formulated, see also this paper for a full list of references. Another discussion and some related problems can be found in [3].

Observations from outside the atmosphere of the Earth during the Skylab missions produced soft X-ray pictures revealing that the corona of the sun is highly structured. One of the features is the presence of a hot plasma confined to quite persistent magnetic loops, see Fig. 1. The longevity of the hot plasma naturally leads to its modelling in terms of a static energy balance between conductive energy transport, heating and radiative losses; see the energy balance equation (1). As the strong magnetic field dominates completely the dynamics of the plasma, the behaviour of the plasma is considered to be one dimensional.

The first term in equation (1) is based on the well established theory of thermal conduction in plasmas, the third term corresponds to calculations of radiative losses in thin, hot plasmas by several authors; see for details [2]. For lack of a consistent theory at present the heating losses are usually represented by a positive constant $\alpha$. In

†Now at: Centre for Mathematics and Computer Science, PO Box 4079, 1000 AB Amsterdam, The Netherlands. 


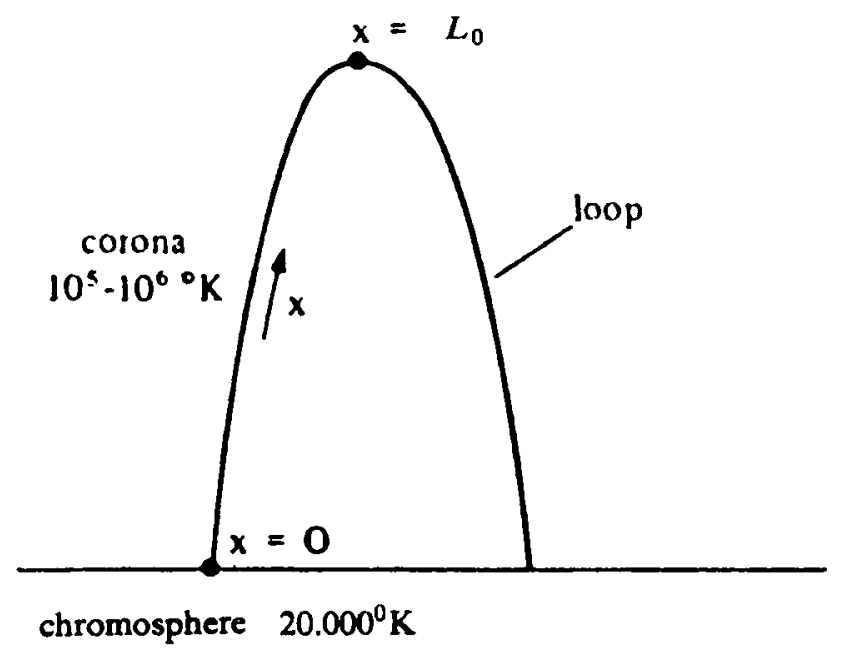

Fig. 1. Coronal loop

the future one would replace this constant by a positive function of $T$; this would not seriously affect the mathematics of this paper.

Equation (2) has been formulated assuming that gravity everywhere is parallel to the magnetic field. This slightly overestimates the influence of gravity at the top of the loop but we note that the plasma is extremely thin at the top. The equations for static solutions become in this way:

$$
\begin{aligned}
& \varepsilon \frac{P}{T} \frac{\mathrm{d}}{\mathrm{d} z}\left(T^{3 / 2} P \frac{\mathrm{d} T}{\mathrm{~d} z}\right)+\alpha-P^{2} \chi(T)=0, \\
& \frac{\mathrm{d} P}{\mathrm{~d} z}=-M y,
\end{aligned}
$$

in which $T$ is the temperature in the loop, $P$ the pressure, both made dimensionless; $z$ is a spatial mass coordinate such that $z=0$ corresponds to the base of the loop, $z=1$ corresponds to the summit. The positive constant $\alpha$ is a dimensionless parameter indicating the heating rate, $\chi(T)$ is the radiative loss function; $M$ is the total mass per unit surface area, $g$ the gravitational constant. The dimensionless parameter $\varepsilon$ is small, $\varepsilon \approx 10^{-2}$ for the solar corona, and is among others proportional to the thermal conductivity of the plasma.

The first constraints are the boundary conditions

$$
T(0)=T_{0}, \quad \frac{\mathrm{d} T(1)}{\mathrm{d} z}=0
$$

with $T_{0}$ a small positive constant. Another constraint is the loop length condition,

$$
\int_{0}^{1} \frac{T}{P} \mathrm{~d} z=L_{0} .
$$

In the case of the solar corona we have $L_{0}=10$.

The static problem, consisting of the equations (1-2) together with the constraints (3-4), is non-standard and no general theory is available. It is interesting to note that 
recently Hulshof et al. [1] proved the existence of monotonically increasing solutions $T(z)$ of problem $(1-4) ; P(0)$ is restricted to a certain range of values. These authors do not exclude the existence of non-monotonic solutions.

In section 2 we shall supplement and correct the analysis in [2] by showing that near the base of the loop there are two adjacent boundary layers where the temperature increases very quickly when moving upward in the loop; in these thin layers the pressure is nearly constant. In section 3 , guided by the asymptotic expansions, we present numerical calculations. Also we show that the type of radiative loss function required in this model triggers off the existence of non-monotonic solutions apart from the monotonic solution found in [1]. This second solution of equations (1-2) with conditions (3-4) bifurcates off the first boundary layer at the base of the loop.

\section{Boundary layers near the base of the loop}

The radiative loss function $\chi(T)$ used in [2] for the solar corona takes the form

$$
\begin{array}{ll}
\chi(T)=\beta_{0} \varepsilon^{-6} T^{5}, & T_{0} \leqslant T \leqslant \tau ; \\
\chi(T)=\beta_{1} T^{-5}, & T_{0} \geqslant \tau .
\end{array}
$$

$\beta_{0}$ and $\beta_{1}$ are constants which are $O(1)$ with respect to the small parameter $\varepsilon$ and which have been chosen such that $\chi(T)$ is continuous; for the solar corona we took as the 'unit' of temperature $2 \times 10^{5} \mathrm{~K}$ and $\tau=0.1$. To give an explicit numerical calculation we shall use this form of $\chi(T)$. We stress however that, as in [1], the analysis applies to a wide range of realistic radiative loss functions.

Near the base of the loop, for $T_{0} \leqslant T \leqslant \tau$, we can start with the analysis in [2] where no boundary layer near $z=0$ has been assumed to exist. They assume that near the base of the loop thermal conductivity plays no part. This means that locally the solution of equation (1) can be approximated by

$$
\alpha-P^{2} \chi(T)=0 .
$$

This relation yields the temperature behaviour

$$
T(z)=\left(\frac{\alpha}{\beta\left(P_{0}-M g z\right)^{2}}\right)^{1 / 5} .
$$

It turns out that the solutions thus obtained do not produce high enough temperatures to be of practical interest. The alternative is that near the base of the loop a thin layer, a boundary layer, exists where we have a steep temperature gradient and therefore a notable thermal conductivity. The appropriate scaling for $T_{0} \leqslant T \leqslant \tau$ is

$$
T=\varepsilon^{v} \tilde{T}, z=\varepsilon^{\sigma} \zeta \text { with } 0 \leqslant v \leqslant 1, \sigma>0 .
$$

Equation (1) takes in this domain the form

$$
\varepsilon^{1+\frac{3}{2} \nu-2 \sigma} \frac{P}{\tilde{T}} \frac{\mathrm{d}}{\mathrm{d} \zeta}\left(\tilde{T}^{3 / 2} P \frac{\mathrm{d} \tilde{T}}{\mathrm{~d} \zeta}\right)+\alpha-\beta_{0} \varepsilon^{-6+5 v} P^{2} \tilde{T}^{5}=0
$$

Equation (2) becomes

$$
\frac{\mathrm{d} P}{\mathrm{~d} \zeta}=-\varepsilon^{\sigma} M g
$$


As $\sigma>0$, the pressure is to first order constant: $P(\zeta)=P_{0}$. In equation (5) a significant degeneration arises if

$$
1+\frac{3}{2} v-2 \sigma=-6+5 v
$$

The terms dominating the energy balance in the boundary layer are conduction and radiation loss, the heating plays no part to first order.

The size of the boundary layer depends on $T_{0}$ and $\tau$. In the case of the solar corona it makes sense to take $v=\frac{1}{2}$, so that $\sigma=\frac{21}{8}$; this means that the size of the boundary layer in space is of order $\varepsilon^{21 / 8}$. Equation (5) reduces in first order to

$$
\frac{1}{\tilde{T}} \frac{\mathrm{d}}{\mathrm{d} \zeta}\left(\tilde{T}^{3 / 2} \frac{\mathrm{d} \tilde{T}}{\mathrm{~d} \zeta}\right)-\beta_{0} \tilde{T}^{5}=0
$$

which can be integrated to produce in the original variables $z$ and $T$

$$
\varepsilon^{7} T^{3}\left(\frac{\mathrm{d} T}{\mathrm{~d} z}\right)^{2}-\frac{4}{17} \beta_{0} T^{17 / 2}=c
$$

with $c$ a constant.

The phase-plane of equation (7) corresponding to the integral (8) is given in Fig. 2.

Solutions of type II (Fig. 2) are not interesting as $T$ decreases, whereas solutions of types I and III are of interest. When the solution $T(z)$ reaches the value $T=\tau$, equation (1) changes its form and the analysis has to be repeated.

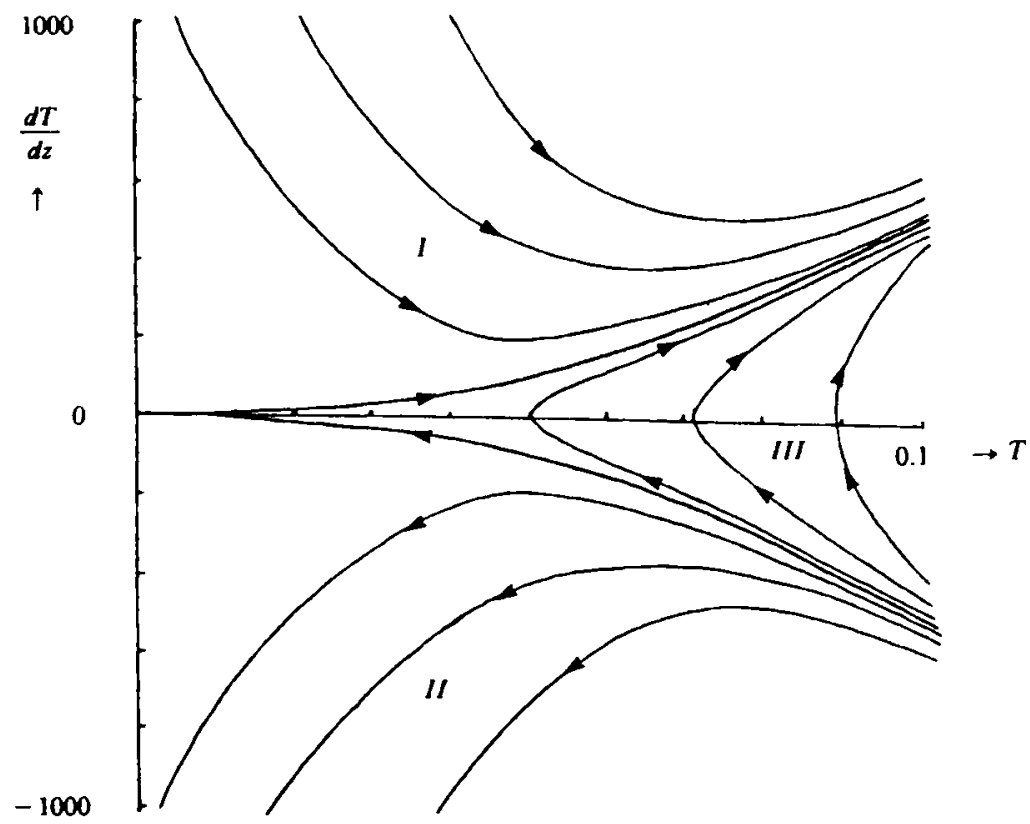

Fig. 2. The $T, \mathrm{~d} T / \mathrm{d} z$ phase-plane in the first boundary layer showing monotonically increasing solutions (I), monotonically decreasing solutions (II) and solutions (III) that initially decrease followed by increase with $z$ 
2.1. The second boundary layer

In the case $T \geqslant \tau$ equation (1) becomes

$$
\varepsilon \frac{P}{T} \frac{\mathrm{d}}{\mathrm{d} z}\left(T^{3 / 2} P \frac{\mathrm{d} T}{\mathrm{~d} z}\right)+\alpha-\beta_{1} P^{2} T^{-5}=0,
$$

$T$ and $z$ are still small so we scale again: $T=\varepsilon^{\nu} \tilde{T}, z=\varepsilon^{\natural} \eta$. Note that we have to take $\mu>0$ as the solution arrives at $T=\tau$ with a steep temperature gradient. The pressure $P$ will still be constant to first order in this second boundary layer for the same reason as before. Equation (9) becomes

$$
\varepsilon^{1+\frac{3}{2} v-2 \mu} \frac{P_{0}}{\tilde{T}} \frac{\mathrm{d}}{\mathrm{d} \eta}\left(\tilde{T}^{3 / 2} P_{0} \frac{\mathrm{d} \tilde{T}}{\mathrm{~d} \eta}\right)+\alpha-\beta_{1} P_{0}^{2} \varepsilon^{-5 v} \tilde{T}^{-5}=0 .
$$

On taking $\tau=0.1$ or $v=\frac{1}{2}$ the radiative loss term still dominates the heating; it is balanced by the conduction if

$$
1+\frac{3}{2} v-2 \mu=-5 v \text { (significant degeneration) }
$$

or $\mu=\frac{17}{8}$. So the second boundary layer is slightly larger, $O\left(\varepsilon^{17 / 8}\right)$ in space. With this choice, equation $(10)$ reduces in first order to

$$
\frac{1}{\tilde{T}} \frac{\mathrm{d}}{\mathrm{d} \eta}\left(\tilde{T}^{3 / 2} \frac{\mathrm{d} \tilde{T}}{\mathrm{~d} \eta}\right)-\beta_{1} \tilde{T}^{-5}=0,
$$

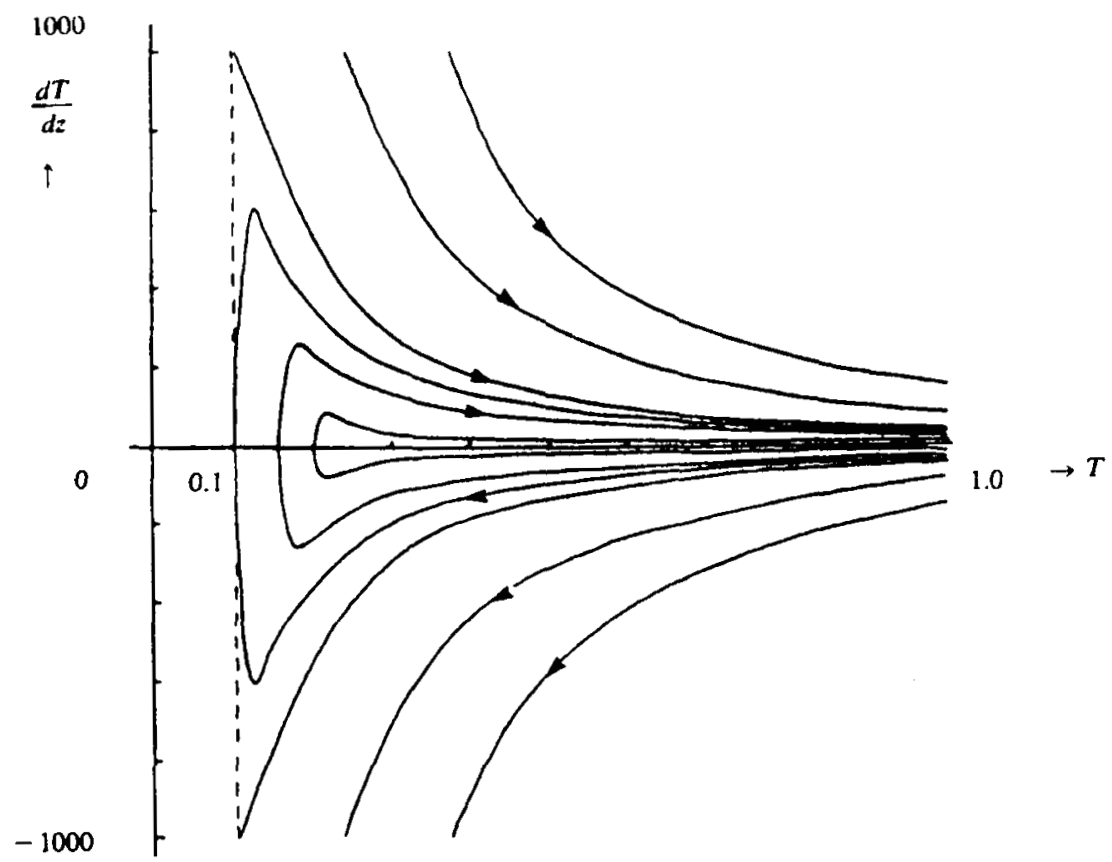

Fig. 3. The $T, \mathrm{~d} T / \mathrm{d} z$ phase-plane in the second boundary layer; solutions start at $T=\tau$ (for the Sun, $\tau=0.1$ ) after which the temperature increases monotonically 
which can be integrated to produce in the original variables $z$ and $T$

$$
\frac{1}{4} \varepsilon T^{3}\left(\frac{\mathrm{d} T}{\mathrm{~d} z}\right)^{2}+\frac{1}{3} \beta_{1} T^{-3 / 2}=d,
$$

with $d$ a constant. The phase-plane of equation (11) corresponding with the integral (12) is given in Fig. 3. We see that, starting with a positive temperature gradient, this quantity remains positive but decreases towards zero.

At the same time the temperature steadily increases until the radiation losses become dominated by the heating. The transition takes place in a neighbourhood of a temperature $T_{\mathrm{h}}$ given by

$$
\alpha=\beta_{1} P_{0}^{2} T_{\mathrm{h}}^{-5} \text {. }
$$

From this stage on, the analysis in [2] (termed there domain III) applies: conduction and heating dominate, there is no spatial boundary layer. The integral describing the behaviour of pressure and temperature is

$$
\varepsilon P^{2}\left(\frac{\mathrm{d} T}{\mathrm{~d} z}\right)^{2} T^{3}+\frac{4}{7} \alpha T^{7 / 2}=e,
$$

with $e$ a constant and $P(z)=P_{0}-M g z$. Equation (14) can be integrated again to produce a relation between $P$ (or $z$ ) and $T$. It follows that the maximum temperature $T_{\max }$ arises at $z=1$ with

$$
T_{\max }=(7 e / 4 \alpha)^{2 / 7} \text {. }
$$

\section{Numerical results}

The ideas developed in the preceding sections are useful in producing numerical results. As in [2] we took values of the constants applicable to the solar corona: $\varepsilon=2.5 \times 10^{-2}, \alpha=0.65, M g=0.4, \beta_{0}=3.81 \times 10^{-4}, \beta_{1}=1.56 \times 10^{-4}, \tau=0.1$. We use a 'shooting' procedure by adding to the boundary values $T(0)=T_{0}$, the value $P(0)=P_{0}$ and the derivative $\mathrm{d} T / \mathrm{d} z(0)$ with large values (from $10^{3}$ to $10^{4}$ ). The solutions of equations (1-2) have to satisfy the boundary value $\mathrm{d} T / \mathrm{d} z(1)=0$ and the loop length condition (4). We find a monotonic solution shown in Fig. 4.

This combined asymptotic-numerical approach can be used to obtain a general insight in the static solutions. We start by omitting the first boundary layer and by considering the solutions from $T_{0}=0.1$ on with a positive temperature gradient.

Equations (1-2) have been solved with this boundary condition at $z=0, \mathrm{~d} T / \mathrm{d} z(1)$ $=0$ and $0.6 \leqslant P_{0} \leqslant 1.76$. This has been carried out by using a stiff ordinary differential equation (ODE) solution from a standard software package; to find the appropriate boundary values at $z=0$ we applied Newton's method. The relation between $P_{0}$ and $\mathrm{d} T / \mathrm{d} z(0)$ corresponding to these solutions has been depicted in Fig. 5 .

Each point of the curve of Fig. 5 corresponds to a solution with different loop length $L_{0}$. In Fig. 6 we give the corresponding loop lengths and, as a quantity of interest, the maximum temperature $T_{\max }$ from equation (15). A lower pressure near $z=0$ requires a higher temperature gradient in the boundary layer near the base of the loop; it produces a longer loop with a correspondingly higher temperature at the summit. 
Coronal Loop Problem

437

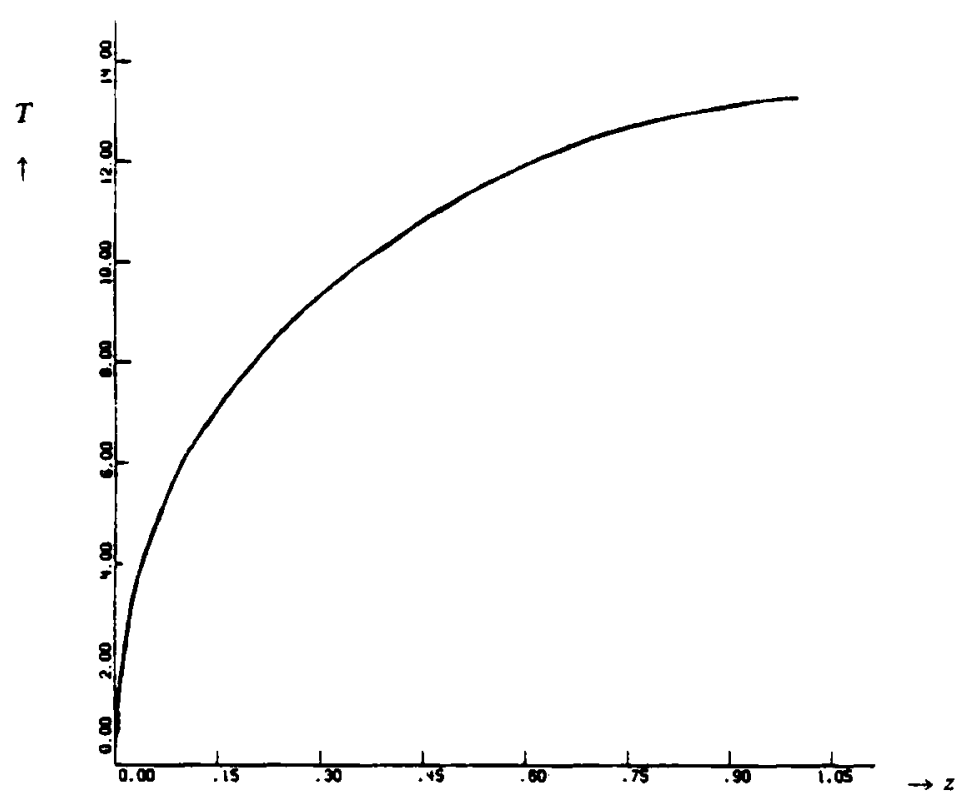

Fig. 4. Monotonically increasing solution $T(z)$ with $T(0)=0.1, P(0)=1.0, \mathrm{~d} T(0) / \mathrm{d} z=11228.59, T_{\mathrm{m}}=13.3$ $\left(\approx 2.66 \times 10^{6} \mathrm{~K}\right), L=13.8\left(\approx 1.3 \times 10^{10} \mathrm{~cm}\right)$

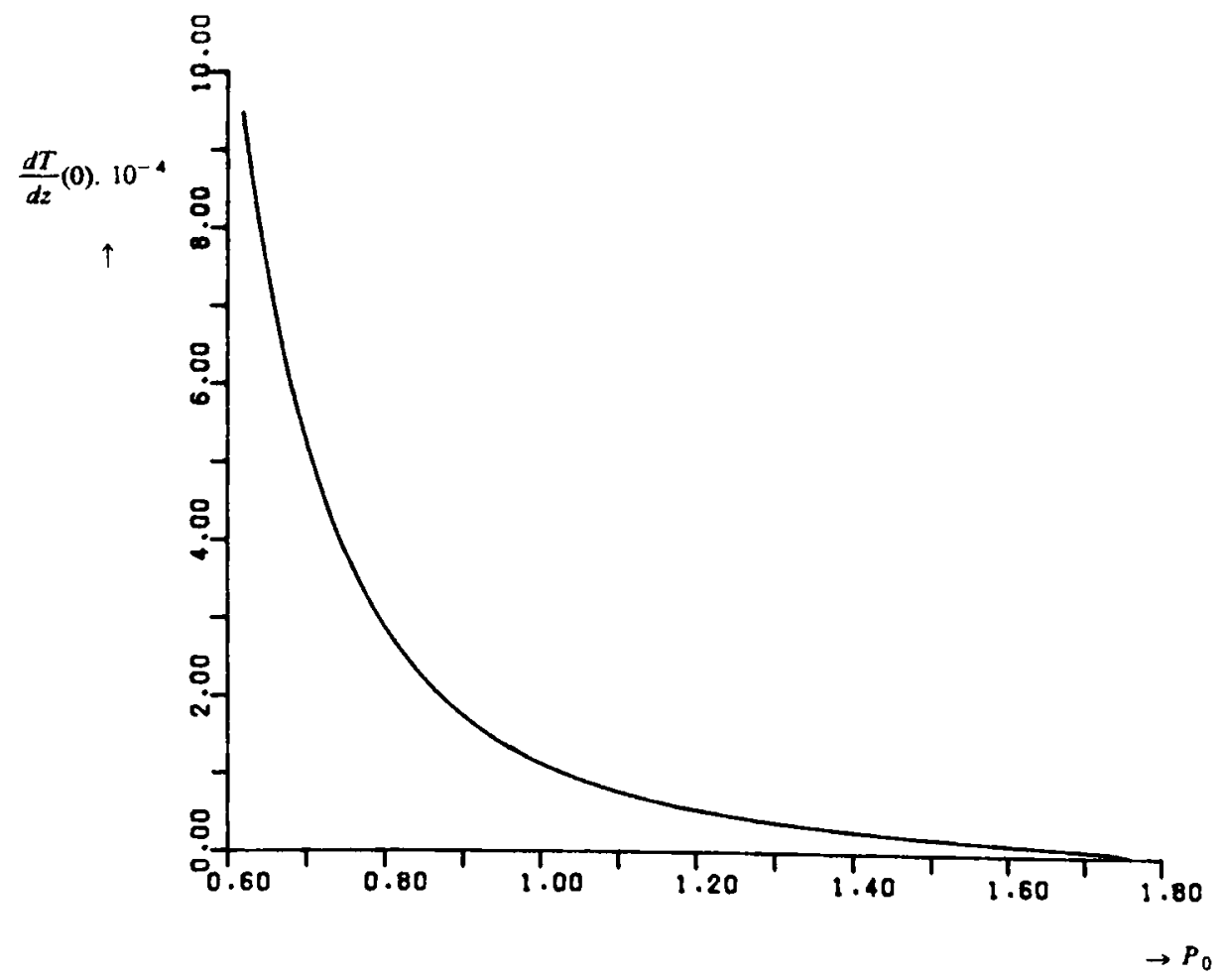

Fig. 5. Relation between $P_{0}$ and $\mathrm{d} T / \mathrm{d} z(0)$ for solutions of equations (1-2) with boundary conditions (3) 

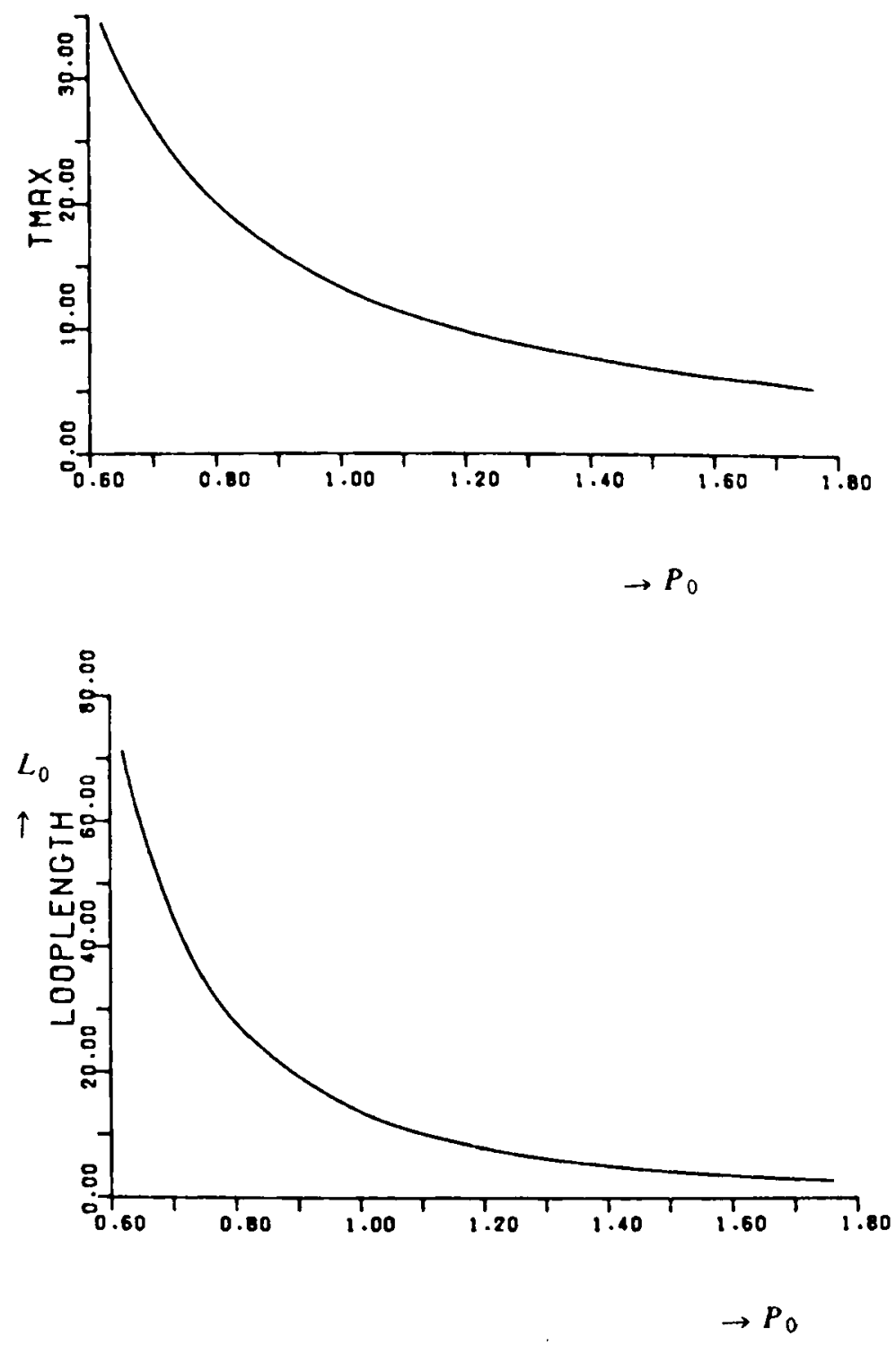

Fig. 6. Maximum temperature $T_{\mathrm{m}}$ and loop length $L_{0}$ as functions of $P_{0}$ for solutions of equations (1-2) and boundary conditions ( 3 )

As a second point of interest we show that we can construct two solutions satisfying equations (1-2) and conditions (3-4). To achieve this we start again with $T_{0}=0.1$ at $z=0$ but with a negative temperature gradient. This puts the solutions in the first boundary layer described in section 1 and, for a range of negative temperature gradients, the solutions are of type III.

From equation (8) we derive that $T$ takes a minimum value,

$$
T_{\min }=\left(-\frac{17}{4} \frac{c}{\beta_{0}}\right)^{2 / 17} \text {. }
$$


Table 1.

\begin{tabular}{lccccccc}
\hline & $T(0)$ & $\mathrm{d} T / \mathrm{d} z(0)$ & $P_{0}$ & $T_{\min }$ & $T_{\max }$ & $L_{0}$ & $\mathrm{~d} T / \mathrm{d} z(1)$ \\
\hline Solution 1 & 0.1 & +277.492 & 1.74988 & 0.1 & 5.3625 & 2.8143 & 0 \\
Solution 2 & 0.1 & -275.429 & 1.75000 & 0.0966 & 5.3636 & 2.8143 & 0 \\
\hline
\end{tabular}

After reaching this minimum value the temperature rises again until $T=0.1$ where $\mathrm{d} T / \mathrm{d} z>0$; from this point on the temperature increases monotonically, resulting in a certain loop length $L_{0}$.

From Figs 5 and 6 we can find a solution that is monotonically increasing for $0 \leqslant z \leqslant 1$ and that satisfies the same conditions (3-4). Thus, we have found two solutions of our problem, which answers a question posed in [1], remark 2.4. As an example we present the parameter values of such a case in Table 1. It is rather surprising that a negative temperature gradient can be changed into a positive temperature gradient in such a small spatial domain as the first boundary layer.

The maximum temperature of the second solution corresponds to $1.07 \times 10^{6} \mathrm{~K}$, the loop length $L_{0}$ to $2.15 \times 10^{9} \mathrm{~cm}$.

An interesting question that remains is the following. If two static solutions exist, it may be expected that at most one of them is stable. Is this the case and which one is stable? This question will be left to future research.

\section{References}

1. Hulshof, J., Terman, D. and Verhulst, F., 'Existence of stationary solutions in the coronal loop problem', Nonlinear Analysis, Theory, Methods and Applications, 13, 841-856 (1989).

2. Pakkert, J. W., Martens, P. C. H. and Verhulst, F., Astron. Astrophys., 179, 285-294 (1987).

3. Verhulst, F., 'Nonlinear diffusion in stellar magnetic loops', Proc. 7th Symp. on Trends in Applications of Mathematics to Mechanics, pp. 35-43, Eds J. F. Besseling and W. Eckhaus, Springer, Berlin, 1988. 